\title{
Enstalé Panno Sou Fénèt ${ }^{1}$
}

Hal S. Knowles, III, Kathleen C. Ruppert, Karla A. Lenfesty, ak Barbara Haldeman²

Zòn ki plis fasil pou resevwa damaj nan siklòn sé Fénèt ki pa pwotéjé. Jan nou te diskité nan dokiman nou-an, Èske Kay nou Paré?, fénèt ki krazégen dwa kite van-an ak lapli rantré nan kay-la é petèt rache do-kay-la.

\section{Ki Sa Ki Bay Fenèt Sékirite?}

Genyen moun ki kole mòso adezif sou vit fenèt pou pwoteje yo. Kole adezif sou fenèt $\boldsymbol{P A}$ fè vit-la pi solid é li $\boldsymbol{P A}$ kapab pwotéjé kay-la si youn bagay frappe-li.

Menm vit ki fèt pou rezisté gwo van gen dwa krazé lè presyon van-an ap pousé sou tout enkadreman fenèt-la. Menm jan-an, papyé plastik éspesyal ke moun mete sou vit-yo pa kapb pwotéjé kay-la tou kont-li. Sèl jan pou pwotéjé fenèt sé enstalé panno.

\section{Kouman Pou Mwen Chyazi Panno Ki Bon Pou Mwen?}

Chak mèt kay fèt pou chwazi panno ki meyè pou li. Bagay pou nou konsidéré sé: Konbyen lajan ke ou genyen, pouki sa ou bezwen-li, konbyen pwoteksyon ou bezwen.

Panno kapab pwotéjé kont bagay kap volé nan lari-a ouswa kont presyon van ap mete sou fénèt, gwo pòt ki genyen vit, pòt a vit ki sou koulis,ak tout lòt ouvèti. Kelke swa ki kalité panno ou chwazi, se jan ou enstalé panno-yo ki pi enpòtan nan zafè pèfòmans.

\section{Lòt Bagay Pou Nou Konsidéré}

Si tout fwa pi gwo pwoteksyon se sa ke ou vlé, fè posib ou pou chwazi pwodwi, fòk ou acheté pwodwi ké Konté Miami-Dade di ke ou kapab itilizé (Gadé nan adrès pwodwi ke nou bay anba-la). Men enstalé panno sèlman pa asé. Li trè enpòtan pou nou itilizé bon jan teknik konstriksyon pou panno-yo kenbé fòs-yo é redwi chans pou bagay pa vole vini frape nou. Bagay pou nou gadé:

- Retiré tout bagay ki deyo nan lakou kankou chèz, tab, bwèt fatra/bwèt resiklaj, plant, etc.

- Mete panno pou pwoteje stikty ankadreman kay-la, $\boldsymbol{P A}$ fenèt ak enkadreman pòt.

- Itilizé matryel ak panno-yo ki pa pe rouyé, kankou panno pou siklòn, vice boulon, etc.

\section{Lòt Resous}

Konté Miami-Dade | Obeyisans Règ Bilding Pou Chèché Pwodwi

http://www.miamidade.gov/buildingcode/onlin e_product_search.asp

NOAA \& AOML | Panno Plywood pou Siklòn http://www.aoml.noaa.gov/hrd/shutters/index2. $\underline{\mathrm{html}}$

1. Dokiman sa-a se pati 2 dé 5 nan Edikasyon + Aksyon = Coleksyon Kontwol Dega Van. Dat piblikasyon: Novanm 2005.

2. Hal S. Knowles, III, Kòdinatè, Pwogram pou Resous Bon Jan Kominoté; Kathleen C. Ruppert, Syantis Estansyon Asosyé, Sévis Estansyon Enèji Laflorid; Karla A. Lenfesty, Espesyalis Kontwol Dega Van Siklòn, Estansyon Kopératif pou Konté St Lucie, Enstiti pou manjé ak Syans Agrikiltirèl, Inivvèsité Laflorid; Barbara Haldeman, Asistan Editè, Sèvis Estansyon Enéji Laflorid.

Enstiti pou manje ak Syans Agrikiltirèl (IMSA) se yon Opòtinité pou Anplwa Egal - Anplwayè Aksyon Afimatif gen dwa pou founi rechèch, enfòmasyon edikasyonèl ak lòt sèvis sèlman bay endividyèl ak enstitisyon kap fonksyoné san fè atansyon a ras, kwayans, koulè, relijyon, laj, enfimité, sex, oryantasyon sexsyèl, stati marital, orijin nasyonal, opinyon ou afilyasyon politik. Pou enfòmasyon sou kouman pou jwenn lòt piblikasyon estansyon, pran kontak ak Biro Sèvis Estansyon Koperatif ki bò Konté pa-ou. Sèvis Estansyon Koperatif Laflorid / Enstiti pou manjé ak Syans Agrikiltirèl / Inivèsité Laflorid / Larry R. Arrington, Dirèktè..

Pwogram Kado nan Depatéman Laflorid sou Afè Kontwol Konstriksyon Rezidans nan Kominoté-a péyé yon pati nan piblikasyon-an. 


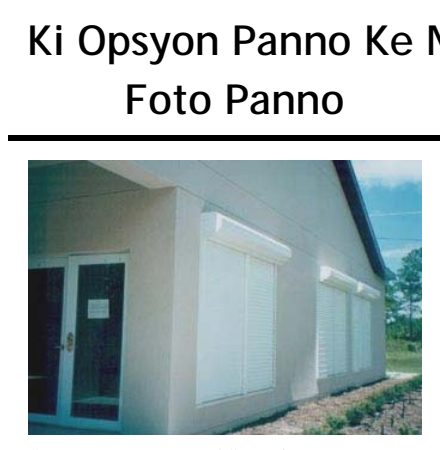

Sous: Estansyon Konté St Lucie

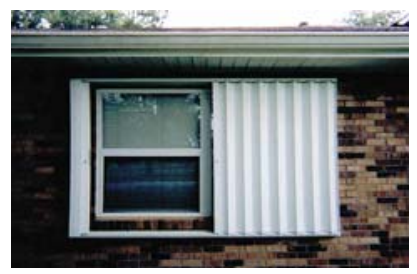

Sous: www.hurricaneshuttercompany.com

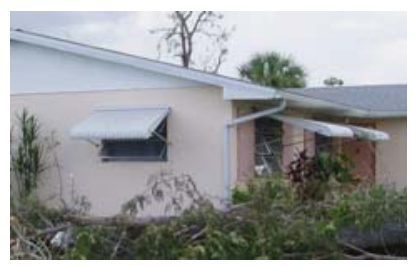

Sous: Pwogram Ki Monitè Kòt Laflorid

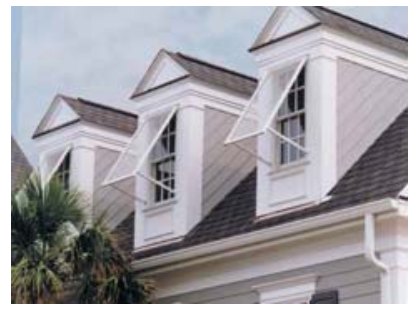

Sous: www.stormshutters.com

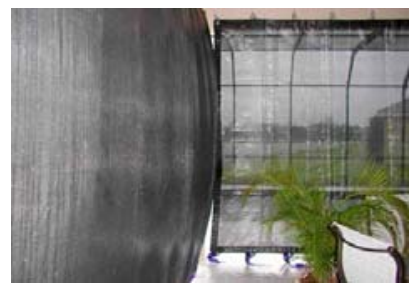

Sous: www.stormshutters.com

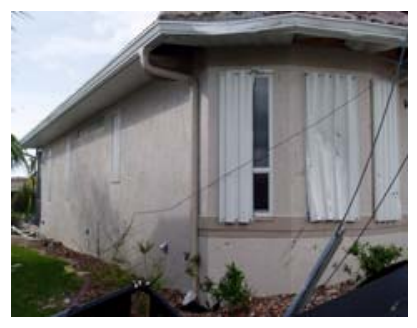

Sous: Pwogram ki Monitè Kòt Laflorid

N/A
Kalite Panno Ak Detay

Woldon Otomatik

Panno sa-yo vini dan youn woulo ki kenbé andedan youn bwèt ki anlè ouvèti ke li sipoze pwotéje-a. Yo fèt jeneralman ak bon jan metal ak kadna anba.

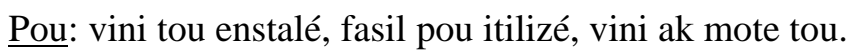

Kont: trè chè.

\section{Akòdeyon}

Yo ralé panno sa-yo sou koté de bò ouvèti ki ap pwotéjé. Yo fèt jeneralman ak bon jan metal ak youn kadna ki kenbé yo ensenm lanmitan ouswa sou youn lòt pyès ki divizé-yo.

Pou: vini tou enstalé, fasil pou itilizé.

Kont: yon ti jan chè, pa twò bel sou yon plan achitèkti.

\section{Ònenn}

Panno sa-yo desann tou dwèt sou miray-la é yo fèmen yo ak kadna sou miray-la. Yo fè yo ak metal ouswa fabèglas é yo bay bon jan lonbraj lè yo pap itilizé-yo.

Pou: vini tou enstalé, fasil pou itilizé.

Kont: yon ti jan chè, pa twò bel sou yon plan achitèkti.

\section{Ònenn Bahama ak Panno Kolonyal}

Ou gen dwa itilizé panno sa-yo kankou Onenn (Ki bay lonbraj lè ou pap itilizé-li) ouswa stil Kolonyal.

Pou: vini tou enstalé, fasil pou itilizé, bel sou yon plan achitèkti.

Kont: yon ti jan chè, Fòs-yo depann de fòm-yo.

\section{Kadran pou Siklòn}

$\$ \$-\$ \$$

Panno sa-yo fèt ak youn twèl sire ki trè solid ki redwi domaj van-an ak fòs enpak debri.

Pou: pa trò chè, kite limyè pasé, fasil pou plyé séré.

Kont: yon ti jan modèn, enpak debri gen dwa krazé vit fenèt ki gen kontak ak kadran-an. Meyè pou ouvèti ki pa genyen vit.

\section{Panno pou Siklòn}

Panno sa-yo fèt pou retire ak enstalé sèlman lè gen siklòn.. Yo fèt en fè, aliminyòm é yo rentré andedan yon trak an metal ki déja enstalé sou miray-la, anba ak anwo ouvèti ke li sipozé pwotéjé-a,ouswa ou kapab monté yo direkteman sou miray-la ak vis.

Pou: bon maché, solid.

Kont: souvan yo lou, difisil pou enstalé é sere.

\section{Plywood}

Gadé dan dokiman-nou-an "Pwotéjé ak Plywood” pou plis Enfòmasyon.
Pri

$\$ \$ \$ \$$

$\$ \$$

$\$ \$$

$\$ \$$

$\$ \$$ 